\title{
High-Efficiency Nanostructuring using Multi-Beam Interference by Consecutively Deposited Ultrashort Laser Pulses on Tool Steel
}

\author{
Chao $\mathrm{He}^{*, * *}$, Michael Steger ${ }^{* *}$, and Arnold Gillner ${ }^{* * * *}$ \\ * Chair for Laser Technology LLT, RWTH Aachen University, Steinbachstrasse 15, Aachen 52074, \\ Germany \\ ** Fraunhofer Institute for Laser Technology ILT, Steinbachstrasse 15, Aachen 52074, Germany \\ E-mail: chao.he@ilt.fraunhofer.de
}

\begin{abstract}
Laser direct patterning by multi-beam interference can create structures in the sub- $\mu \mathrm{m}$ regime on a wide variety of materials. As the structuring process relies on the interference of 2 or more coherent beams, the reachable dimension of a single structure can be comparable to the applied laser wavelength and the structure is replicated simultaneously over the whole interference volume. To obtain a stable interference pattern a classic approach namely spot-by-spot is adopted. In this approach, several laser pulses are deposited at each position on tool steel and there is no pulse overlap between two neighbored pulse positions. Since the surface is patterned spot by spot, a typical average processing rate of the spot-by-spot approach for a submicron periodicity on tool steel is $21 \mathrm{~mm}^{2} / \mathrm{min}$. Aiming to structuring a large scale surface on tool steel with high efficiency and homogenous morphology, we present a patterning strategy, in which laser pulse are deposited with a constant consecutive pulse overlap. In this paper, two patterning methods and periodic nanostructures fabricated by both methods are investigated. As a demonstrator, a $140 \times 140 \mathrm{~mm}^{2}$ area with structural periodicity of $690 \mathrm{~nm}$ is fabricated on tool steel by using consecutively overlapped deposition of interfering ultrashort laser pulses. A processing rate of $360 \mathrm{~mm}^{2} / \mathrm{min}$ can be achieved with a single spot size of $120 \mu \mathrm{m}$ and laser fluence of $0.42 \mathrm{~J} / \mathrm{cm}^{2}$. The productivity of continuous structuring can be enhanced by two orders of magnitude comparing with the classic spot-to-spot approach. In addition, we characterized the local and long range morphological homogeneity of the structured surface by means of quantitative analysis of periodicity and depth of patterned nanostructures.
\end{abstract}

DOI: 10.2961/jlmn.2018.01.0001

Keywords: laser micro processing, multi-beam interference, ultrashort pulsed laser, structural homogeneity, energy deposition, tool steel.

\section{Introduction}

Structures in sub-micron or nanometer scale on the surface of modern devices have the potentials to generate new functions or enhance their mechanic, optical or biological performance ${ }^{[1-4]}$. A variety of application examples have given evidences: thin film structured electrodes for can improve the efficiency of organic light emitting diodes (OLEDs), nanostructured surface on medical components is able to decrease the bacterial attachment and improve the bio-compatibility of implants ${ }^{[5]}$. In order to fabricate such structures, current lithographic techniques such as photolithography, interference lithography, nanoimprint lithography and deep X-ray lithography have been introduced and are considered to be extreme high intricate in terms of equipment design and working conditions e. g. with hazardous materials ${ }^{[6]}$. Furthermore, due to essential multi step processing, the lithographic techniques are time consuming and lack of flexibility. Over last decades ${ }^{[6-10]}$, laser direct writing technique, which allows a high level of flexibility, has been intensively studied and developed. By means of that, the texturization of micro-scaled structures in variety of substrates can be achieved. To fulfil the aim of increasing the resolution and downscaling the structure, multi-beam interference (MBI) with ultrashort pulsed laser is employed. In this pro- cess, the laser intensity distribution is modulated in interference pattern. Moreover, the heat affected zone in the patterning can be minimized by taking advantage of ultrashort laser pulse raditation ${ }^{[11]}$. One of the current challenges in laser multi-beam interference is to obtain interference patterns with high long range homogeneity and smaller periodicity. Bieda et al. fabricated periodic patterns with $0.8 \mu \mathrm{m}$ pitch in stainless steel, copper and titan surface by two interfering laser beams irradiated by a pulsed Yb:YAG Laser at a wavelength of $515 \mathrm{~nm}$ and a pulse duration of $35 \mathrm{ps}^{[6]}$. Another research issue of this technique focuses on the productivity for a large-scaled area, such as a surface of industrial casting dies $^{[5]}$. Lasagni et al. ${ }^{[8,12,13]}$ stated to achieve a structuring speed of about $0.1 \mathrm{~m}^{2} / \mathrm{min}$ with spatial periodicity ranging from 2.2 to $3.1 \mu \mathrm{m}$ in metals using a galvanometric scanner. However, the results of pattern structures on aluminum sample showed an inhomogeneous and droplet covered surface. And the pattern on stainless steel with $1 \mu \mathrm{m}$ period had a structure depth of only approximately $35 \mathrm{~nm}$, which showed a low aspect ratio and structure contrast.

In our previous work ${ }^{[10]}$, laser multi-beam interference (MBI) using a spot-by-spot patterning strategy has been employed to achieve periodic nanostructures with high structural contrast and resolution within the range of single laser spot. Patterning speed has a lower order of priority in the 
fabrication of unique prototypes. However, the requirement on productivity in industrial applications especially those with large scale surface becomes ever higher. The limitation of process speed is dependent on laser performance like available laser pulse energy, coherent length, as well as ablation threshold of materials. In this work, we present laser nanostructuring of tool steel by MBI with an ultrashort pulsed UV laser. Instead of a classic spot-by-spot strategy, we fabricated a large scale continuous line-like nanostructure with a periodicity of $690 \mathrm{~nm}$ by using consecutively overlapped laser pulse deposition. To investigate the local and long range morphological homogeneity of the nanostructure, patterns fabricated by both approaches are observed and analyzed under confocal laser scanning microscope (LSM) and atomic force microscope (AFM).

\section{Experimental setup}

The interference setup for fabrication of periodic nanostructure with a diode pumped Nd:YVO4 MOPA laser (Hyper Rapid, Coherent) is shown in Fig 1. The laser beam source emits a $355 \mathrm{~nm}$ wavelength UV irradiation with a pulse duration of $15 \mathrm{ps}$ and maximum energy $60 \mu \mathrm{J}$. For both structuring strategies, the laser operates at the fundamental frequency of $400 \mathrm{kHz}$, and the output pulse frequency is controlled by setting the duty cycle ratio of laser. The incident beam is firstly focused by a $\mathrm{f}=500 \mathrm{~mm}$ aspheric focusing lens and then split by a diffractive optical element (DOE). Apart from the -1 and +1 order, which contain $78 \%$ of the incident laser power, all other orders that come out from DOE are blocked by a filter. The two coherent beams are reflected by a HR coated prism to two tunable mirrors, thus the incident angle $\alpha$ of two laser beams on the sample can be adjusted for varied pattern periodicity. In this experiment, the incident angle was set to $10^{\circ}$ which equals a periodicity of approximately $1.3 \mu \mathrm{m}$. As sample tool steel X37CrMoV5 with a hardness of $57 \pm 1 \mathrm{HRC}$ was used and placed on a high resolution XY moving stage (Planar DL, Aerotech), which is capable of achieving $1 \mathrm{~m} / \mathrm{s}$ velocities and a straightness down to $\pm 0.4 \mu \mathrm{m}$. The experiments were conducted with a laser spot size of $120 \mu \mathrm{m}$ on the sample surface. The morphology of irradiated samples was analyzed by means of a confocal laser scanning microscope (LSM, VK-9700, Keyence) and atomic force microscope (AFM, Rados N8, Bruker).

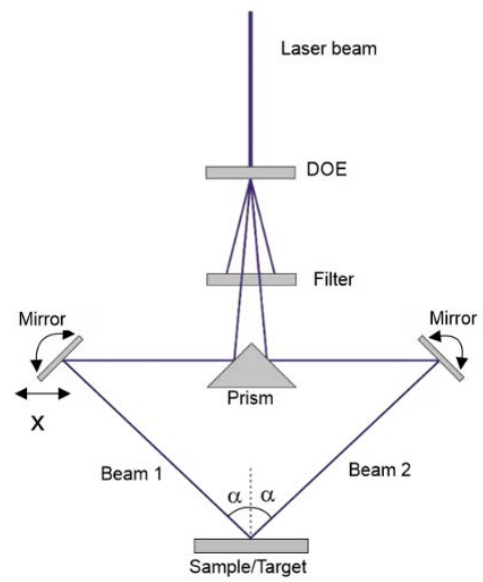

Fig. 1 Schematic diagram of the experimental setup for the multibeam interference (MBI)
To characterize the homogeneity on a large-scale patterned surface, 16 points with an area of $15 \times 15 \mu \mathrm{m}^{2}$ in an arrangement of a 4 by 4 array were randomly selected, where 4 points locate in a row in the direction parallel to the orientation of interference pattern. The distances between areas in a row are nearly $25 \mathrm{~mm}$. Periodicity and depth of the pattern at each point are measured and analyzed by AFM.

\section{Results and discussion}

\subsection{Pulse energy deposition for the two structuring strategies}

A classic approach namely spot-by-spot strategy is employed for patterning the nanostructures on tool steel. In this process, multiple laser pulses with optimized laser fluence are deposited at one position. The multiple laser pulses are necessary in order to, on one hand, avoid redundant heat effect induced by a single laser pulse with equivalent fluence, on the other hand to achieve aimed structure depth by adequate laser energy deposition where the pulse fluence is slightly higher than the ablation threshold of material. The characteristic of this method is a static superposition of laser pulses which contributes to a precise and stable ablative behavior. The laser beam is not moved during the ablation of the single spot and larger areas need to be stitched together from these single spots as shown in Fig. 2a.

In order to avoid a positioning error in large scale area and utilize the thermal incubation in ablation process, the multiple laser pulses are successively deposited in one position. Therefore, a dwell/pause of motion stage must be calculated between the locations of two neighbored spot in one direction. Owing to this, high frequent acceleration and deceleration of axis is unavoidable. As a result, the structuring efficiency is decreased. A typical average process rate of spot-by-spot method was $3 \mathrm{~mm} / \mathrm{s}$ for 10 pulses per spot.

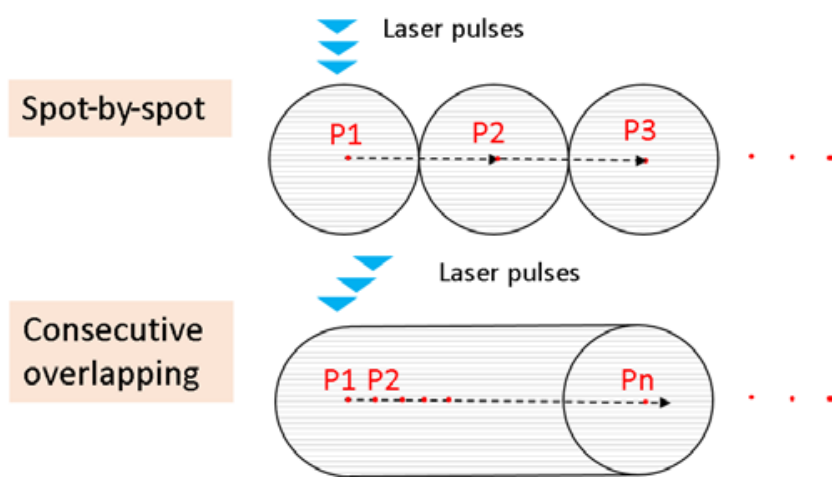

Fig. 2 Illustration of pulses position in approaches of classic spotby-spot and consecutively overlapping

In contrast to a static superposition of laser pulses in spot-by-spot approach, a structuring strategy by using consecutively overlapped laser pulses on linear path during structuring is introduced. The arrangement of pulse position in this dynamic processes is illustrated in Fig. 2b.. To guarantee a well-defined periodic structure, the scanning direction is chosen to be parallel to the orientation of interference pattern.

An overlap degree $\eta_{\text {overlap }}$ of laser pulses is introduced to quantify and represent the density of laser pulses deposited on the impact surface. It is given by: 


$$
\eta_{\text {overlap }}=1-\frac{v_{s} / f}{d_{w}+v_{s} \cdot t_{p}}
$$

Where, $v_{s}$ is scanning speed of axis, $f$ is applied laser frequency, $d_{w}$ refers the beam intensity $1 / \mathrm{e}^{2}$ diameter of laser spot and $t_{p}$ is pulse duration.

Considering the application of an ultrashort laser pulses, $t_{p}=15 p s$, the overlap degree of can be given by:

$$
\eta_{\text {overlap }}=1-\frac{v_{s}}{d_{w} \cdot f}
$$

With processing parameters: scanning speed of $100 \mathrm{~mm} / \mathrm{s}$, laser spot size $120 \mu \mathrm{m}$ and applied laser frequency $10 \mathrm{kHz}$, an overlap degree of $91.6 \%$ is yielded. In addition, an overlapping of $20 \mu \mathrm{m}$ between patterning tracks is set in the consecutive pulse deposition method.

\subsection{Morphological analysis of sub-micrometer-struc- ture}

To describe the homogeneity of the structured surface, filling factor and contrast of periodic structure are studied. As schematically demonstrated in Fig. 2, a higher filling factor of interference patterns up to a theoretic value $100 \%$ can be achieved by consecutive laser pulses due to that the marginal area between circular laser spots can be filled by a high overlapping degree. To obtain a more intuitive overview on the patterned areas by the two pulse deposition strategies, optical microscopic images were recorded under a magnification of 300 times and are shown in Fig. 3.

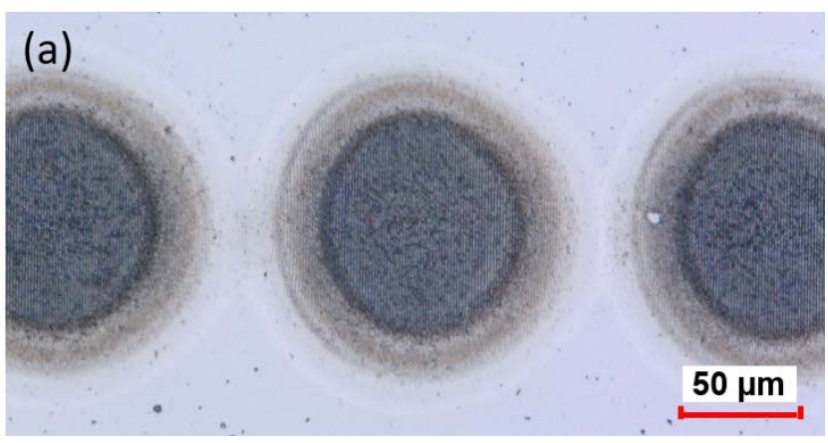

(b)

\section{$50 \mu \mathrm{m}$}

Fig. 3 Patterned areas by using spot-by-spot (a) and consecutively overlapped pulses (b)

Fig. 3a shows the allocation of the three single spots deposited in chronological order. The border of each spot can be clearly detected due to the ablative process and unstructured areas exist among deposited laser spots. The Fig. 3b shows a structured area with a path overlap of $20 \mu \mathrm{m}$ instead of a single line. Compared with the pattern in Fig. 3a, the surficial homogeneity of the area structured by consecutive pulses in Fig. 3b has been significantly enhanced. A minimized thermal effect at the identical applied laser fluence of
$0.11 \mathrm{~J} / \mathrm{cm}^{2}$ is apparently to be seen in Fig. 3b. Owing to energy diffusion by consecutively deposited laser pulses at 10 $\mathrm{kHz}$ repetition rate, the local thermal accumulation decreases on the structuring path.

An additional observation of the samples in Fig. 3 was carried out under a laser scanning microscope with a $1500 \mathrm{X}$ magnification to investigate the homogeneity of nanostructure within a pulse spot fabricated by MBI using spot-byspot (Fig. 4a) and consecutively overlapped laser pulses (Fig. 4b). As shown in Fig. 4a, the pattern from center to rim of laser spot is inhomogeneous in regard of structure contrast. It is evident that, the periodic structure at the rim of spot is shallower than at the center which is attributed to the Gaussian distribution of the laser intensity. In contrast to that, the structured area in a long range in Fig. $4 \mathrm{~b}$ is uniform and the line-like pattern is well-defined. Oxidized layer covered surface, which was caused by redundant laser energy deposition in spot-by-spot method, cannot be detected in Fig. 4b
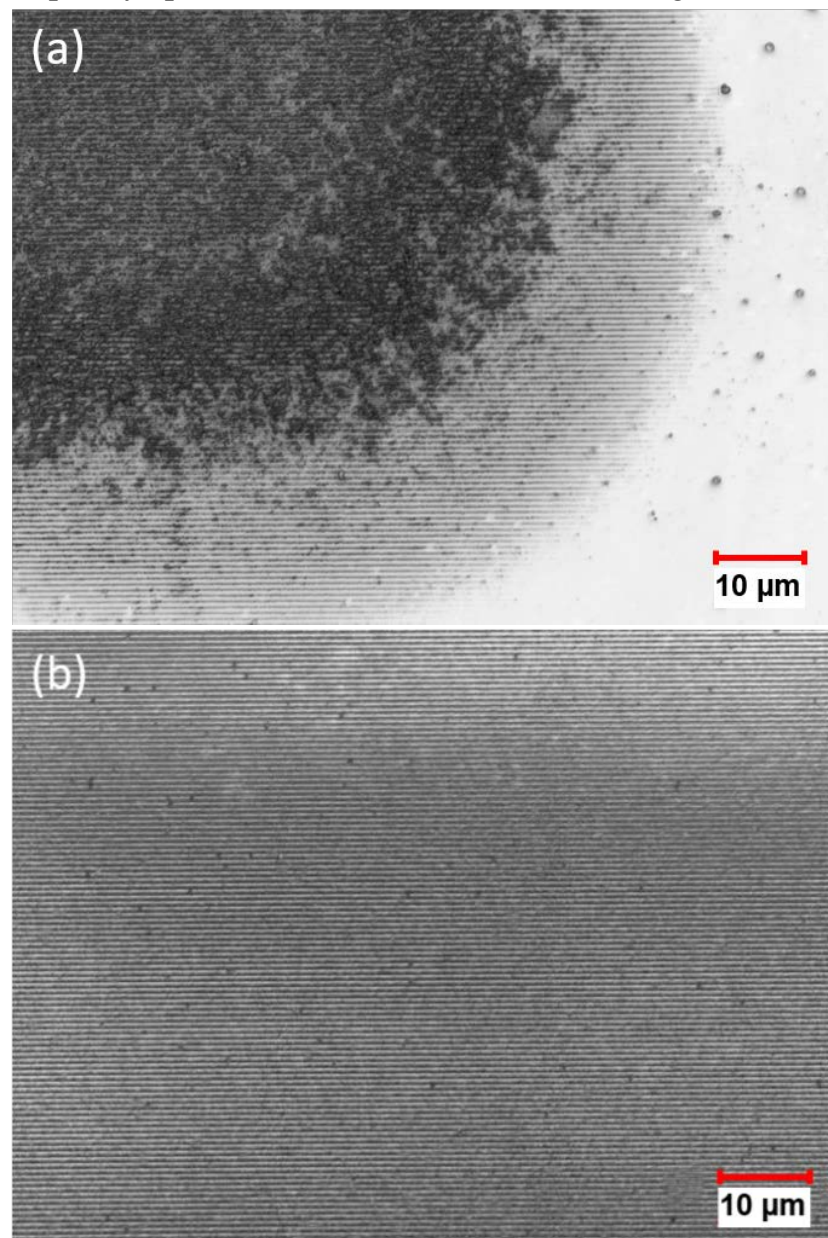

Fig. 4 Laser scanning microscope image of line-like fabricated by means of spot-by-spot (a) and consecutive nanostructuring (b)

To measure the aspect ratio of the line-like structures by both methods, its periodicity and depth were measured by an atomic force microscope (AFM). The 3D profiles and measurements are shown in Fig. 5. A $10 \mu \mathrm{m}$ by $10 \mu \mathrm{m}$ area in the center of the spot/line including 10 structure elements was captured in the AFM image shown in Fig. 5. An average periodicity of $1.3 \mu \mathrm{m}$ and depth of $0.75 \mu \mathrm{m}$ are derived from the measured profile in Fig. 5a. Similarly, the interference pattern in Fig. 5b, which fabricated by consecutive pulses overlapping method, has an average periodicity of $1.3 \mu \mathrm{m}$ and a depth of $0.18 \mu \mathrm{m}$. 
(a)
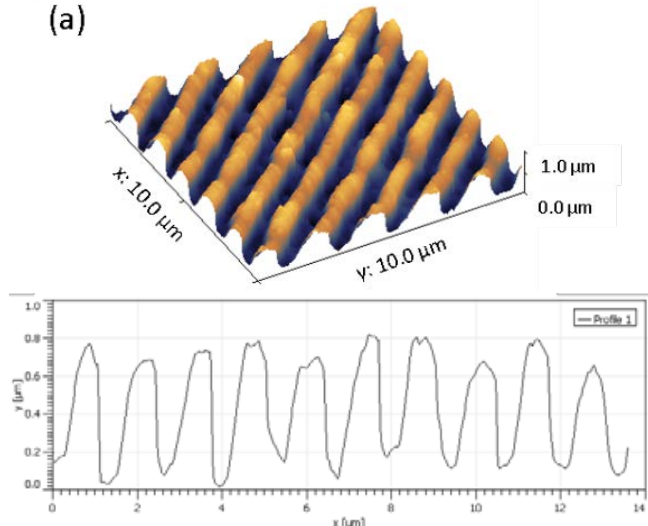

(b)
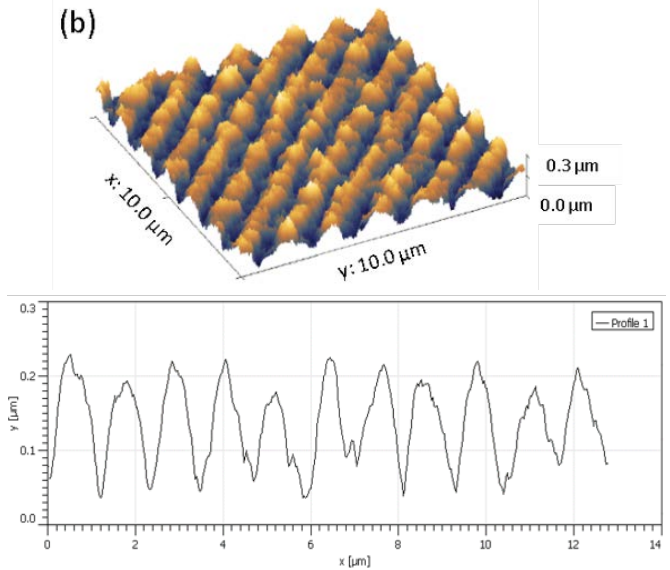

Fig. 5 Atomic force microscope (AFM) measurements on the morphology of line-like periodic nanostructures. (a) spot-by-spot and (b)consecutive overlapping

It is evident from Fig. 5 that the structural aspect ratio with consecutively overlapped laser pulse deposition is lower than that of static pulse deposition. However, based on the further investigation on the development of depth of periodic nanostructures in the direction parallel to the orientation of interference pattern, nanostructures by consecutively overlapped pulse deposition demonstrates a more stable behavior as shown in Fig. 6. It represents a local homogeneity of the patterns, which has a fluctuation of approximately $15 \mathrm{~nm}$ of a mean depth. While the structure depth of the pattern fabricated by spot-by-spot approach decreases dramatically from center to the rim of a laser spot due to a Gaussian distribution of laser intensity in the laser spot.

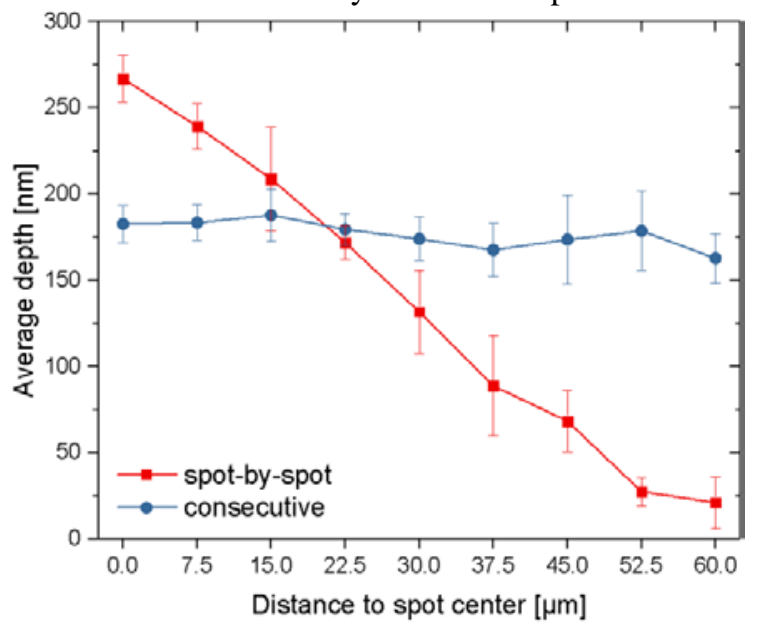

Fig. 6 Averange depth of periodic nanostructures within the range of half of a laser spot fraom center to rim in the direction parallel to the orientation of interference pattern

\subsection{Characterization of morphological homogeneity for nanostructuring on large scale surface by consecu- tively overlapped pulse deposition}

With the aim of demonstrating the rapid nanostructuring for large scale area by multi-beam interference, A 140 x 140 $\mathrm{mm}^{2}$ tool steel with $\left(\mathrm{Cr}_{1-\mathrm{x}} \mathrm{Al}_{\mathrm{x}}\right) \mathrm{ON}$ hard coating for injection molds was structured by means of consecutive pulses overlapping method. A fabrication rate of $360 \mathrm{~mm}^{2} / \mathrm{min}$ at a laser fluence $0.42 \mathrm{~J} / \mathrm{cm}^{2}$ was achieved. The interference patterns in neighbored squares have perpendicular orientations, the local structural diffraction colors of one orientation can be only observed at a certain observation angle. Thus, two alternating colors (light and dark) can be distinguished. To decrease the lateral periodicity of MBI patterned structures and raise its resolution, one simple way is to increase the incident angle of two coherent laser beams. Fig. 7 shows periodic nanostructures with a grating periodicity of $690 \mathrm{~nm}$. For that, an inclination angle of $15^{\circ}$ was set.

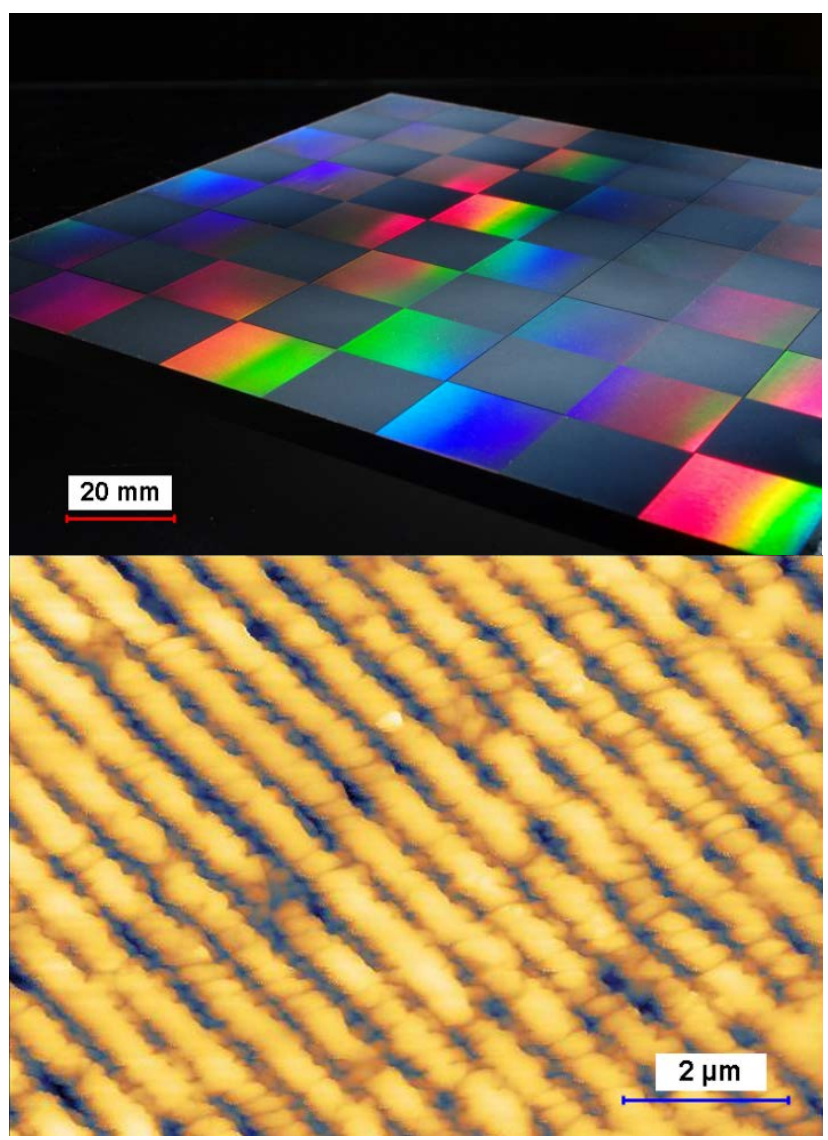

Fig. 7 Multi-beam interference nanostructuring of a chess board on a $\left(\mathrm{Cr}_{1-\mathrm{x}} \mathrm{Al}_{\mathrm{x}}\right) \mathrm{ON}$ hard coated tool steel for injection molds and AFM image of periodic nanostructures.

In the process of multi-beam interference using consecutively overlapped laser pulse deposition, the stability of periodicity and depth of patterns can be affected by several factors such as mechanical vibration and laser power instability. The morphological homogeneity of large-scale structured pattern on the demonstrator, therefore, is characterized by a quantitative analysis on periodicity and structural depth of interference pattern. Fig. 8a and 8b present the scatter diagrams of the measurement of periodicity and structural depth at 16 different points. The diagram in Fig. 8a gives evidence that the global periodicity is very stable over a large surface, the average periodicity is $690 \mathrm{~nm}$ with a standard deviation 
of $2.57 \mathrm{~nm}$, which is in measurement tolerance. Whereas the curve of depth in Fig. 8(b) indicates a larger deviation over a long range. However, a much smaller deviation is shown in the patterned structures at four points in a row (Position 1-4, 5-8. 9-12 and 13-16). As aforementioned a depth difference in the pattern occurs in the spot owing to a Gaussian distribution of the laser intensity. Perpendicular to the direction of the orientation of the interference pattern, the superposed laser intensity is still a Gaussian distribution in spite of a path overlap of $20 \mu \mathrm{m}$ is applied.
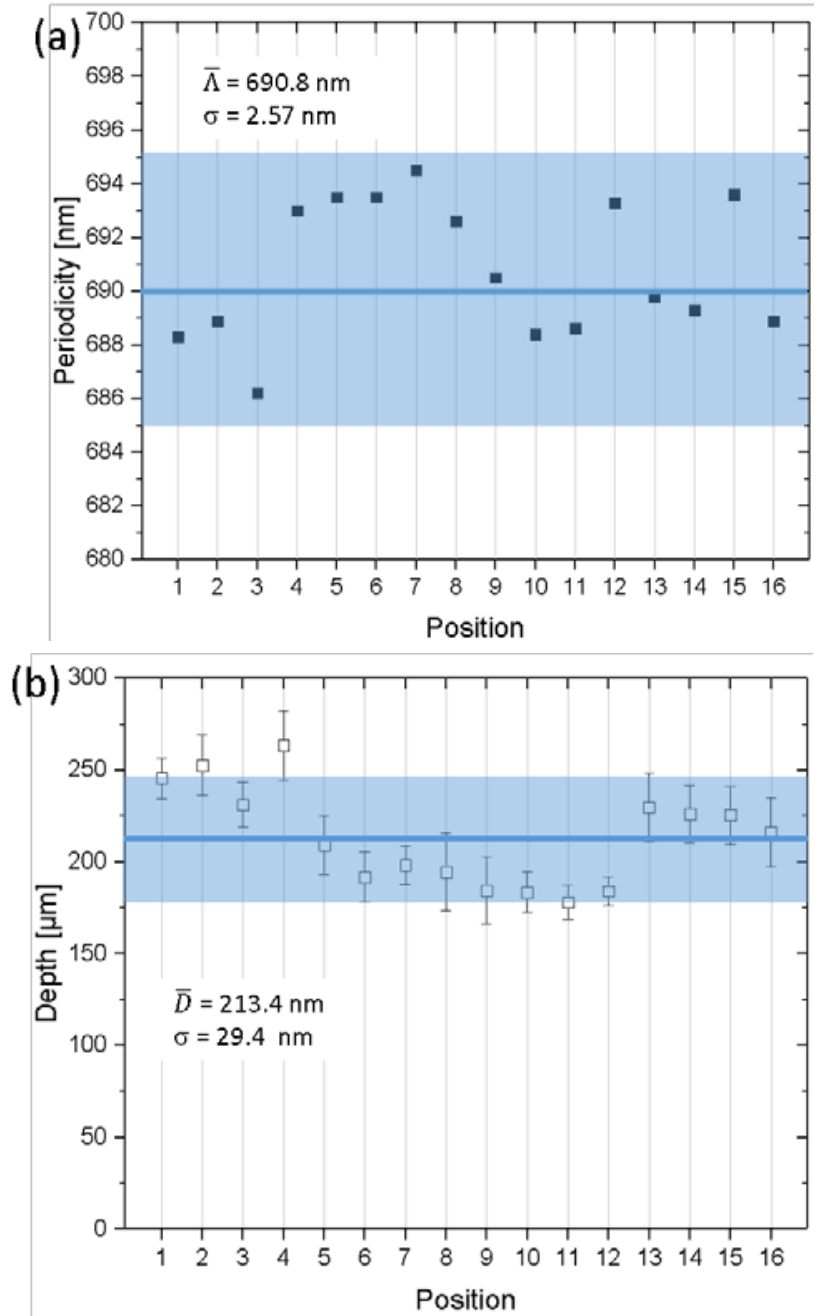

Fig. 8 Scatter diagrams of periodicity (a) and depth of nanostructures at different position on a large-scale surface patterned by consecutively overlapped laser pulses

\section{Conclusion}

Multi-beam interference with ultrashort pulse laser was applied to a tool steel sample with consecutively overlapped pulse strategy to increase the fabrication speed and enhance the long range homogeneity of the patterned surface. Compared with classic spot-by-spot patterning approach, this structuring strategy is characterized as a dynamic process without a dwell in the motion axis for deposition of single pulse or pulse train. Firstly, the fabrication speed can be increased to $100 \mathrm{~mm} / \mathrm{s}$ and the throughput can be scaled up by two orders of magnitude. Secondly, a $100 \%$ fill factor on sample surface can be achieved owing to a high overlapping rate of laser pulsed in scanning directing and between paths. And the whole structured area has a higher uniformity with well-defined nanostructure compared to the spot-by-spot strategy.

When the incident angle of coherent laser beam is increased to $15^{\circ}$, nanostructuring with a periodicity of $690 \mathrm{~nm}$ can be fabricated on a large scale surface of a (Cr1-xAlx)ON hard coated tool steel. For a further enhancement of the fabrication efficiency together with morphological quality in tool steel, applied laser fluence should be adapted with scanning speed as well as effective working spot size. The way of laser energy deposition plays a vital role in periodic nanostructuring using MBI for high resolution and high homogeneity patterns in industrial applications.

As a final remark, the characterization of the long range homogeneity of nanostructures pattern by MBI by quantitative analysis on the periodicity and depth indicate a stable process using consecutively overlapped laser pulse deposition.

\section{Acknowledgments}

The depicted research was funded by the Deutsche Forschungsgemeinschaft (DFG) as part of the program Cluster of Excellence "Integrative Production Technology for Highwage Countries” at RWTH Aachen University.

\section{References}

[1] G. M. Burrow and T. K. Gaylord: Micromachines, 2, (2011) 221.

[2] J. Berger, T. Roch, S. Correia, J. Eberhardt and A. Lasagni: Thin Solid Films, 612, (2016) 342.

[3] Z. Zhou, Z. Song, L. Li, J. Zhang and Z. Wang: Appl. Surf. Sci., 335, (2015) 65.

[4] M. F. Broglia S. Suarez, F. Soldera, F. Mücklich, C. A. Barbero, R. Bellingeri, F. Alustiza and D. Acevedo: Appl. Surf. Sci., 300, (2014) 86.

[5] A. Lasagni, T. Roch, M. Bieda, D. Benke and E. Beyer: Proc. of SPIE, 8968, (2014) p.89680A-1

[6] M. Bieda, M. Siebold and A. Lasagni: Appl. Surf. Sci., 387, (2016) 175.

[7] B. Voisiat, M. Gedvilas, S. Indrišiūnas and G. Račiukaitis: J. Laser Micro/Nanoeng., 6, (2011) 185.

[8] M. Bieda, E. Beyer, and A. F. Lasagni: J. Eng. Mater. Technol., 132, (2010) 031015.

[9] A. Lasagni, P. Shao, J. Hendricks, C. Shawe, D. Martin, and S. Das: Appl. Surf. Sci., 256, (2010) 1708.

[10] M. Steger, C. Hartmann, S. Beckemper, J. Holtkamp and A. Gillner: J. Laser Micro/Nanoengin., 8, (2013) 210.

[11]R. Poprawe: "Tailored Light 2-Laser Application Technology” ed. by Springer (Publisher, Heidelberg, 2011) p. 351.

[12]A. Lasagni, D. Benke, T. Kunze, M. Bieda, S. Eckhardt, T. Roch, D. Langheinrich and J. Berger: J. Laser Micro/Nanoengin., 10, (2015) 340.

[13] V. Lang, T. Roch and A. Lasagni: Adv. Eng. Mater., 18, (2016) 1342. 